\title{
Time Perspectives and Delay of Gratification - The Role of Psychological Distance Toward the Future and Perceived Possibility of Getting a Future Reward
}

This article was published in the following Dove Press journal: Psychology Research and Behavior Management

\author{
Sue-Jung Kim $\mathbb{D}^{\prime}$ \\ Hyo-Jeong $\mathrm{Kim}^{2}$ \\ Kiho $\mathrm{Kim}^{3}$ \\ 'Department of Psychology, Chung-Ang \\ University, Seoul, South Korea; \\ ${ }^{2}$ Department of Psychology, Seoul \\ National University, Seoul, South Korea; \\ ${ }^{3}$ Department of Psychology of \\ Counseling, Sejong Cyber University, \\ Seoul, South Korea
}

Purpose: This study investigated how an individual's time perspective of the present and the future affects the delay of gratification, using the construal level theory. In addition, the mechanisms that influence the time perspective on the delay of gratification were examined through the mediating roles of the psychological distance and the perceived possibility of getting a future reward.

Participants and Methods: One hundred twenty university students completed the Korean version of the Swedish Zimbardo Time Perspective Inventory (S-ZTPI) and performed a Temporal Discounting task to aid in the evaluation of their ability to delay gratification. Their psychological distance to the future and perceived possibility of getting a future reward were measured using the visual analogue scale.

Results: The results showed that once the Present-Hedonistic and Future-Negative from among the six-time perspectives increased, and the ability to delayed gratification decreased. On the other hand, once the Future-Positive time perspective increased, the ability to delayed gratification increased. Only the psychological distance for 9 months was associated with time perspective and the mediation effect was not significant. Present-Hedonistic time perspective negatively predicted the perceived possibility of getting a future reward and the delay of gratification. The perceived possibility of getting a future reward fully mediated the relation between the Future-Negative time perspective and the delay of gratification.

Conclusion: These findings suggest that problems involved with the delay of gratification (such as smoking, addiction, and binge eating behavior) are more likely to occur in people who have high Present-Hedonistic and Future-Negative time perspectives, because these time perspectives lead to a lower perceived possibility of getting a future.

Keywords: time perspective, delay of gratification, construal level theory, psychological distance, perceived possibility of getting a future reward

\section{Introduction}

Time seems to be given equally to all, but in reality, people perceive subjectively. In other words, people have different "Time Perspectives (TP)", meaning implicit or explicit attitudes toward time. The TP is an unconscious process whereby individuals determine their preferences from among past, present and future time perspectives. $^{1,2}$ The Zimbardo Time Perspective Inventory (ZTPI) and Swedish Version of the Zimbardo Time Perspective Inventory (S-ZTPI) are the most widely used scales to measure the difference in peoples' TP. ${ }^{3,4}$ The S-ZTPI identifies the six distinct components of an individual's attitudes toward time. The Past-Positive
Correspondence: Kiho Kim

Department of Psychology of Counseling, Sejong Cyber University, Cheonho-Daero 680, Gwangjin-Gu, Seoul 04992, South Korea

Email kihohere@gmail.com 
time perspective (PP) embodies a nostalgic or positive attitude toward the past, and the Past-Negative time perspective (PN) is characterized by a pessimistic, negative construction of the past. The Present-Hedonistic time perspective (PH) is characterized by an impulsive attitude and orientation toward present enjoyment, while the PresentFatalistic time perspective (PF) is characterized by a hopeless and helpless perspective regarding time. The Future-Positive time perspective (FP) is related to social motivation and the achievement of future goals, and the Future-Negative time perspective (FN) includes negative expectations and worry about future goals. Although each TP has its own distinct characteristics, an individual's TP cannot be determined by only one TP. Multiple TP can coexist within one individual. ${ }^{5}$

Recent studies regarding TP have focused on how differences in TPs can influence the lives of individuals. Researchers suggest that a balanced TP may be related to adaptive functioning and wellbeing. ${ }^{5-8}$ Preoccupation with a specific time frame can result in a temporal bias and is related to serious problems like the failure to delay gratification, substance abuse and gambling., ${ }^{3,9-12}$ In particular, delay of gratification is closely associated with TP. ${ }^{13-16}$ Delay of gratification refers to the delay of immediate, smaller gratification for the sake of profit from more distant but deferred goals. ${ }^{17}$ The ability to delay gratification is a protection factor against negative interpersonal relationships and personal vulnerabilities, and its defects can be an important variable causing addiction behavior. ${ }^{18}$ The success and failure of sustaining the delay of gratification are determined by a value of reward and effort to control the behavior. The delay of gratification will succeed if the value of the reward is evaluated greater than the effort spent in controlling the behavior, and vice versa. ${ }^{19}$ Since the subjective value of a reward is reduced as a function of time, time is an important factor when assessing the value of a reward between two choices. ${ }^{20}$

It has been empirically reported that disorders with difficulty in the delay of gratification, such as addiction disorder, are closely related to Present-Hedonistic and Future time perspectives. ${ }^{21-23}$ Specifically, the PresentHedonistic time perspective is associated with deficits in the ability to delayed gratification, and Future time perspective is related to success in that. Addicts that are present-oriented have a shorter time perception, in that they could consider only 9 days into their future. ${ }^{24-26}$ Therefore, they tend to act more impulsively and rarely think about potential negative consequences that could result from the action. ${ }^{27}$ On the other hand, individuals with long-term time perception have longer-term plans for the future and exhibit more successful delay of gratification. $^{28,29}$ Researches showed higher futureoriented TP predict less addictive behaviors and healthier behaviors. ${ }^{12,30}$

However, there is no known mechanism by which the TP affects the delay of gratification so far although the relationship between TP and the delay of gratification has been widely researched. Further information regarding the features of delay of gratification is needed in order to understand how it can be affected by the TP. According to the model developed by Reynolds and Schiffbauer to help understand the delay of gratification, ${ }^{19}$ success or failure in the delay of gratification could be influenced by the perceived value difference between the choice options and the perceived differential probability of actually receiving a reward. Especially important to the delay of gratification is how the person ideates the rewards for which they are waiting, or the perceived value of the delayed reward. ${ }^{17,31}$ The value of the delayed rewards depends on the amount of the reward and length of the delay. ${ }^{32}$ Therefore, the perceived value of the delayed reward would be determined by the amount of the reward and the perception of the delay length. While the amount of the reward is fixed, the perception of the length of delay is an important factor in the delay of gratification, since the perception of the length of delay is subjective.

To be more specific, the perception of the length of delay could be explained by the temporal psychological distance of the Construal Level Theory (CLT), considering that the same periods are perceived differently by each person. ${ }^{33}$ Psychological distance refers to the level of psychological proximity to the time or events. ${ }^{34}$ According to the CLT, the different psychological distances from the future affect the way individuals behave and make decisions by changing their mental representation of the future. ${ }^{34,35}$ High-level abstract construal is used to represent psychologically distant distances, while lowlevel concrete construal is used to represent psychologically close distances. High-construal of the distant future is likely to be more abstract and simple, while low-level of the near future is likely to be more concrete and complex. ${ }^{33}$ Likewise, the same future event will look different from the different psychological distance. ${ }^{33,35}$ Therefore, if the psychological distance toward the future increases, the future will feel like a long time and the value 
of the reward in the future will decrease because the reward seems abstract. In actual fact, vivid imagination of future events has been shown to increase the delay of gratification in several studies. $^{27,36,37}$

In addition, success or failure in the delay of gratification would be influenced by the perceived possibility of getting a future reward. The perceived possibility of getting a future reward could be different depending on one's view of life. Individuals with a negative view of life have an anxious tendency to take immediate rewards, because the reward may become unavailable over time. Individuals with a positive view of life tend to think that future rewards are available, so they consider future rewards to be worth the wait. $^{38,39}$

As can be understood from above, assessing the perception of the length of delay and the perceived possibility of getting a future reward plays an important role in the success or failure to delay of gratification. TP refers to the orientation and attitude toward time, so it seems to be a concept that includes both the perception of the length of delay and the perceived possibility of getting a future reward. In other words, the time orientation of the TP has been associated with the accessibility of distant future construal (future-oriented TP) or temporal psychological distance (present-oriented TP).

Also, the perceived possibility of getting a future reward is linked with the attitude toward the time of the $\mathrm{TP}$, particularly positive and negative attitudes about the future.

The aim of the study is to find out the effect of TP on the delay of gratification. Although the relationship between TP and the delay of gratification has been widely researched, the characteristics of the $\mathrm{TP}$ are important factors in determining the delay of gratification. Additionally, most of the studies have been conducted using a questionnaire, and we empirically investigated the effects of TP on the delay of gratification using a temporal discounting task to compensate for this limitation.

Another aim of this study is to determine the mechanism by which the TP affects the delay of gratification, using the psychological distance and the perceived possibility of getting a future reward. Although there are many studies on the relationship between TP and the delay of gratification, there is no known mechanism by which the TP affects the delay of gratification, to date. In this study, we will directly measure the psychological distance and perceived possibility of getting a future reward, and whether the TP actually relates to these characteristics. Building on the findings of relevance between the TP and the psychological distance, as well as the perceived possibility of getting a future reward, we hope to determine the mechanism by which the TP affects the delay of gratification.

In summary, our hypotheses can be presented as follows:

1. Present-Hedonistic and Future-Negative perspectives are negatively related to the delay of gratification (ie, choosing small rewards at present).

2. The Future-Positive perspective is positively related to the delay of gratification (ie, choosing greater rewards in the future).

3. The psychological distance will mediate the relationship between the TP and delay of the gratification.

4. The perceived possibility of getting a future reward will mediate the relationship between the TP and the delay of gratification.

\section{Methods}

\section{Participants}

A total of 120 undergraduate students from Chung-Ang University participated in this study. We removed 16 outliers, using Cook's D distance measure. ${ }^{37}$ The mean Cook's D for the total sample $(\mathrm{N}=120)$ was 0.009 . We used the guideline of $\mathrm{D}>4 / \mathrm{N}$, where $\mathrm{N}$ is the number of observations. $^{38}$ The data of sixteen participants with greater values than $0.03 \quad(=4 / 120)$ were eliminated. Therefore, we are reporting data from a final sample of 104 students. None of the participants reported any physical or psychological problems, and their state of health was checked by an interview. This study was approved by the Institutional Review Board of Chung-Ang University (IRB No. 1041078-201508-HR-130-01) and with the Declaration of Helsinki. All subjects signed informed consent.

\section{Materials}

\section{Swedish Version of the Zimbardo Time Perspective Inventory (S-ZTPI)}

The S-ZTPI measures individual differences in timeorientation and attitudes. ${ }^{4}$ The S-ZTPI contains 64 items that measure six subscales: Past-Positive (PP), PastNegative (PN), Present-Hedonistic (PH), Present- 
Fatalistic (PF), Future-Positive (FP), and Future-Negative (FN). The FP and FN were added in the S-ZTPI as an extension to the ZTPI, ${ }^{3}$ which included one future dimension. Specifically, PastNegative includes 10 items (4, 5, $18,24,31,37,38,42,56,61$ ) such as, "I think about the bad things that have happened to me in the past," PastPositive includes 9 items $(2,7,12,17,22,28,33,46,55)$ such as, "It gives me pleasure to think about the past," Present-Hedonistic includes 15 items (1, 9, 13, 19, 21, 26, $29,31,35,36,47,50,52,54,63)$ such as, "Taking risks keeps my life from becoming boring," Present-Fatalistic includes 9 items $(3,15,39,42,43,44,53,59,60)$ such as, "Fate determines much in my life", Future-Positive includes 11 items $(6,11,14,23,27,34,45,49,51,58$, 62) such as, "When I want to achieve something, I set goals and consider specific means for reaching those goals", and Future-Negative includes 10 items $(8,10,16$, $20,24,32,40,48,56,64)$ such as, "The future contains too many boring decisions that I do not want to think about." The S-ZTPI has been translated from the source into the target language. The translation and back translation of the original S-ZTPI were conducted by professional interpreters. The participants responded using a 5-point scale, ranging from 1 (very uncharacteristic) to 5 (very characteristic). The mean scores for each subscale were calculated for use in the analysis. The internal consistency of S-ZTPI is adequate (Cronbach's $\alpha=0.78$ ). The Cronbach's $\alpha$ of each subscale is as follows: PP (0.71), PN (0.84), PH (0.74), PF (0.74), Future-Oriented (0.77).

\section{Temporal Discounting Task}

The temporal discounting task was used to measure the delay of gratification ability with respect to hypothetical amounts of money. ${ }^{40-43}$ Previous studies using hypothetical reward produced results comparable with outcomes using real monetary amounts. ${ }^{44,45}$ In this study, we used the temporal discounting task applying a double-limits algorithm. $^{42,44}$ For this task, short instructions were given by the experimenter. The instructions stated that a series of choice slides would be presented and the participants were asked to make choices between a smaller amount that could be received immediately and another larger amount that could be received after a delay. Participants were asked to exclude certain factors such as their financial situation. They were told to try only to select the preferred option. The temporal discounting task was run using E-Prime version 2.0. Choices were between a variable immediate amount
$(<100,000$ won; worth approximately $\$ 100)$ or 100,000 won (worth approximately $\$ 100$ ) with six delays (a week, 3 weeks, 2 months, 5 months, 9 months, and 18 months). ${ }^{42,43}$ Both immediate and delayed amounts were presented together and the participants selected the left or right option in the slide using keys on the keyboard. If participants preferred the left side option, they pressed the 1 key and if they preferred the right-side option, they pressed the $2 \mathrm{key}$. The sides on which the immediate and delayed rewards were presented were counterbalanced, with the first choice of 50,000 won now vs 100,000 won later. For the next choices, the immediate reward was varied based on the participant's previous choices. The values of the immediate choices were recalculated using the double limits algorithm. ${ }^{44}$ If the participants chose the immediate rewards, the algorithm reduced the immediately available amounts. However, if the participants choose the delayed rewards, the algorithm increased the immediately available amounts. Choice slides ended once indifference points had been determined for the reward corresponding to each delay period. Indifference points mean immediate amount value at which choices of larger amount 100,000 won at a given delay were equally likely. There were individual differences in the number of questions needed to determine the indiscriminate amount, because the responses vary from person to person. In this study, an average of 124 questions was presented.

\section{Psychological Distance Toward the Future and Perceived Possibility of Getting a Future Reward}

A Visual Analogue Scale (VAS) was used to assess the psychological distance toward the future and the perceived possibility of getting a future reward. In this study, psychological distance refers to the level of psychological proximity to the future. The perceived possibility of getting a future reward refers to how much people believe that they will get a reward in the future. It is linked to the specific choices that were being made. The questions used were: "How far away is the psychological distance to the future (6 delays later: a week, 3 weeks, 2 months, 5 months, 9 months, and 18 months) reward?", and

What are your thoughts on the chances of getting the future reward when you have chosen reward in the task (6 delays later: a week, 3 weeks, 2 months, 5 months, 9 months, and 18 months)? 
These contain 6 items ( 6 delays) each. The VAS consisted of a $100 \mathrm{~mm}$ horizontal line (0: very close; 100: very far away, or 0: not at all; 100: very much). The VAS about the psychological distance toward the future (Cronbach's $\alpha=$ 0.90 ) and the perceived possibility of getting a future reward (Cronbach's $\alpha=0.91$ ) has good internal consistency.

\section{Barratt Impulsivity Scale (BIS)}

Since there are a lot of evidence that impulsivity plays an important role in delay of gratifications, we controlled the impulsivity trait of the individual in the analysis. ${ }^{9,41}$ The BIS is a self-report scale developed to measure impulsivity by asking the subject to answer questions about the ways they act and think at the usual time. ${ }^{46}$ The Korean version of BIS has 23 questions, each of which required the respondent to choose from 1 (rarely, never) to 4 (almost always). The BIS has good internal consistency in this study (Cronbach's $\alpha=0.81$ ).

\section{Economic Level}

Because the delay discounting task about money is used in this study, economic levels of participants can affect the choice between an immediate reward and delayed reward. So, we tried to control the economic level, which was measured on a VAS. The questions used were: "What do you think your economic status?", measuring the economic level subjectively perceived by the individual. The VAS consisted of a $100 \mathrm{~mm}$ horizontal line (0: Extremely low, not very good; 100: Extremely high, very good).

\section{Procedure}

All of the participants volunteered for the experiment via either an online system or a class and were tested individually. Participants completed the S-ZTPI before attending the experiment to reduce the demand characteristics of S-ZTPI on choice between future and immediate rewards. When each participant arrived at the laboratory, they signed an informed consent form, and then completed the demographics questionnaire. Next, the participants were given instructions regarding the task, which stated that a series of choice slides would be presented, and that they would be asked to make choices between a smaller amount that could be received immediately and another larger amount that could be received after a delay. When the participants finished reading the instructions, the temporal discounting task was initiated. The temporal discounting task was presented, and the responses were recorded on a computer. The participants then reported their psychological distance using the VAS. Upon completion, the participants were debriefed and were given a gift coupon (worth approximately \$5) as a reward. The experiment took approximately 30 minutes to complete.

\section{Data Analysis}

All statistical analyses were performed using SPSS 17.0 for Windows. Before using regression analysis, determination of non-violation of assumptions of correlation and multiple regressions were performed. In addition, the centering was applied to minimize multicollinearity because some TPs are highly correlated with each other. ${ }^{47}$ The hierarchical multiple linear regression analysis was performed to identify which predictors accounted for the most unique variance in the delay of gratification. The hierarchical multiple linear regression was examined for multivariate outliers using Cook's D distance measure. ${ }^{48}$ The ability to delayed gratification was estimated using an area-under-the-curve (AUC) analysis, which has been suggested as a theoretically neutral measure of discounting. ${ }^{49}$ The AUC was calculated to plot the indifference points against time and ranged from 0 to 1 . The higher the AUC, the lower the rate of discounting which means a good delay of gratification. Finally, we conducted additional analyses to test the proposed mediation models using Hayes' PROCESS macro for SPSS. ${ }^{50}$ We ran PROCESS's mediation model 4 with 5000 bootstrapping.

\section{Results}

\section{Sample Characteristics}

After screening participants for the exclusion criteria, 104 students ( 67 females, 37 males; age: $M=22.36, S D=$ $2.60)$ remained. The descriptive sample characteristics and main study variables are presented in Table 1 .

\section{The Effects of Time Perspectives on Delay of Gratification}

In order to identify which predictors accounted for the most unique variance in the delay of gratification, impulsivity and economic levels were entered into the first step of the regression, and the six TP (Past-Positive, Past-Negative, PresentHedonistic, Present-Fatalistic, Future-Positive, and Future-Negative) were entered into the second step of the hierarchical multiple linear regression analysis (Table 2). A significant increase in the variance between the first step of the regression and the second step $\left(\Delta R^{2}=0.29\right)$ suggests that the TP significantly predicted the delay of gratification 
Table I Sample Characteristics

\begin{tabular}{|l|l|}
\hline Variable & Sample Statistics \\
\hline $\begin{array}{l}\text { Age (years) } \\
\text { Mean (SD) }\end{array}$ & $22.36(2.60)$ \\
\hline Sex (\%) & \\
Men & 35.6 \\
Women & 64.4 \\
\hline Impulsivity & \\
Mean (SD) & $2.20(0.35)$ \\
\hline Perceived Economic Level & \\
Mean (SD) & $53.56(16.72)$ \\
\hline Time Perspectives (M, SD) & \\
Past-Positive & $2.91(0.64)$ \\
Past-Negative & $3.69(0.53)$ \\
Present-Hedonistic & $3.33(0.52)$ \\
Present-Fatalistic & $2.64(0.57)$ \\
Future-Positive & $3.46(0.54)$ \\
Future-Negative & $3.09(0.45)$ \\
\hline
\end{tabular}

after controlling for the other variables (impulsivity and economic level). As seen in Table 2, all of the TPs emerged as statically significant predictors of the delay of gratification, with the exception of Present-Fatalistic. Past-Positive ( $\beta$ $=0.27, p<0.01)$, Past-Negative $(\beta=0.36, p<0.01)$, and Future-Positive $(\beta=0.29, p<0.05)$ were all positively associated with the delay of gratification. These results mean that once Past-Positive, Past-Negative, and FuturePositive increase, the ability to delayed gratification ability increases. In contrast, Present-Hedonistic $\beta=-.55, p<$ $0.001)$ and Future-Negative $(\beta=-.20, p<0.05)$ emerged as significant inverse predictors of the delay of gratification. These results mean that once Present-Hedonistic and FutureNegative increase, the ability to delayed gratification ability decreases. The four most influential factors were PresentHedonistic, Past-Negative, Future-Positive, Future-Negative.

\section{The Relationship Among Time Perspectives, Psychological Distance, and Delay of Gratification}

To explore the relationships between the TP and psychological distance, a correlation analysis was applied. And then a meditation analysis was applied to test our hypotheses 3 and 4. All TPs were not associated with the psychological distance. Only the psychological distance for 9 months showed positive correlations with PresentHedonistic and Future-Negative time perspectives $(r=$ $0.27, r=0.20$ ), and negative correlation with FuturePositive time perspective $(r=-.23)$. Also, the results demonstrated that the psychological distance did not predict the delay of gratification, so the mediation effect was also not significant.

\section{The Relationship Among Time Perspectives, Perceived Possibility of Getting a Future Reward, and Delay of Gratification}

The result of path analysis (Figure 1) showed that PresentHedonistic time perspective negatively predicted the perceived possibility of getting a future reward $(\beta=-.23, p<$ $0.05)$ and the delay of gratification $(\beta=-.49, p<0.001)$. Future-Positive time perspective positively predicted the delay of gratification $(\beta=0.51, p<0.001)$, but it was no longer a significant predictor for the perceived possibility of getting a future reward ( $\beta=0.09$, n.s., see Figure 2). Future-Negative time perspective negatively predicted the perceived possibility

Table 2 Analysis of Hierarchical Regression for the Prediction of Delay of Gratification

\begin{tabular}{|c|c|c|c|c|c|c|c|c|c|}
\hline Model & Variables & B & SE & $\boldsymbol{\beta}$ & $\mathbf{t}$ & $\mathbf{p}$ & $\mathbf{R}^{2}$ & $\operatorname{AdjR}^{2}$ & $F(p)$ \\
\hline \multirow[t]{2}{*}{ I } & Impulsivity & -1182.79 & 294.76 & -0.37 & -4.01 & $<0.001$ & & & $9.01(<.001)$ \\
\hline & Economic Level & 9.08 & 6.25 & 0.13 & 1.45 & 0.15 & 0.15 & 0.14 & \\
\hline \multirow[t]{8}{*}{2} & Impulsivity & 36.92 & 402.88 & 0.01 & 0.09 & 0.93 & 0.44 & 0.4 & $9.25(<.001)$ \\
\hline & Economic Level & 7.25 & 5.53 & 0.11 & 1.31 & 0.19 & & & \\
\hline & Past-Positive & 578.07 & 199.84 & 0.27 & 2.9 & $<0.01$ & & & \\
\hline & Past-Negative & 630.94 & 184.91 & 0.36 & $3.4 I$ & $<0.01$ & & & \\
\hline & Present-Hedonistic & $-|219.6|$ & 292.1 & -0.55 & -4.18 & $<0.001$ & & & \\
\hline & Present-Fatalistic & 182.33 & 212.38 & 0.09 & 0.86 & 0.4 & & & \\
\hline & Future-Positive & 611.65 & 234.58 & 0.29 & 2.61 & $<0.05$ & & & \\
\hline & Future-Negative & -485.25 & 241.9 & -0.2 & -2.01 & $<0.05$ & & & \\
\hline
\end{tabular}




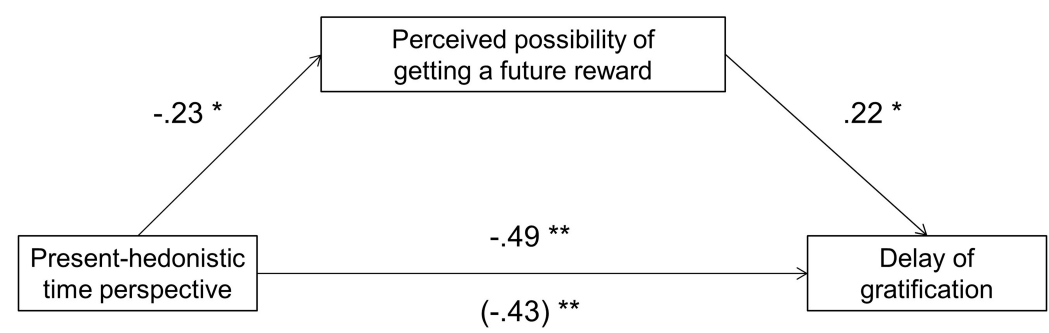

Figure I Standardized regression coefficients for the pathways among present-hedonistic time perspective, perceived possibility of getting a future reward, and delay of gratification. Notes: $* p<0.05$; $* * p<0.001$.

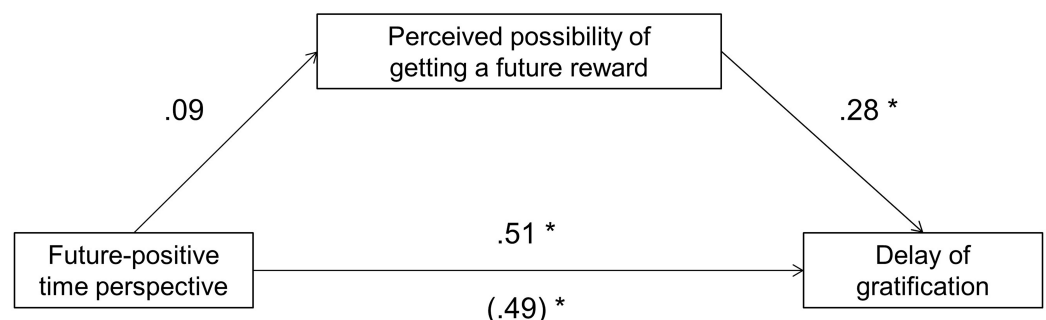

Figure 2 Standardized regression coefficients for the pathways among future-positive time perspective, perceived possibility of getting a future reward, and delay of gratification. Note: $* p<0.001$.

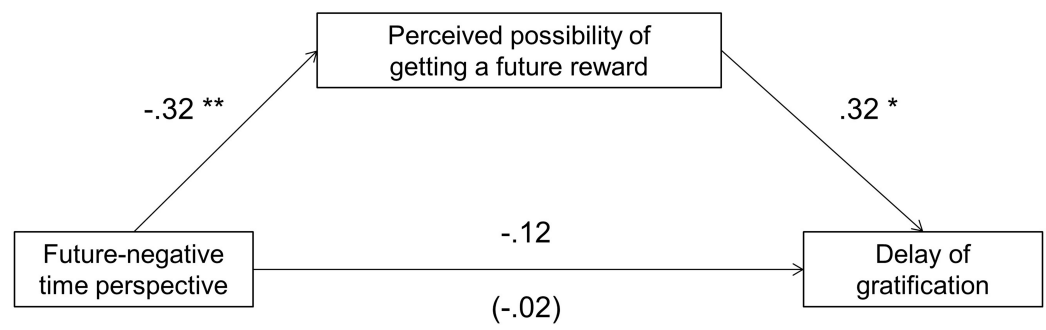

Figure 3 Standardized regression coefficients for the pathways among future-negative time perspective, perceived possibility of getting a future reward, and delay of gratification. Notes: ${ }^{*} p<0.01$; ${ }^{* *} p<0.001$.

of getting a future reward $(\beta=-.32, p<0.001)$, but did not predict the delay of gratification $(\beta=-.12$, n.s., See Figure 3$)$.

A mediation analysis showed an indirect effect of the Present-Hedonistic time perspective on the delay of gratification via the perceived possibility of getting a future reward of 0.06 , bootstrapped $95 \%$ CIs [-.130, -.001], SE $=0.03$, indicating a significant partial mediation. Also, the direct effect Present-Hedonistic time perspective on the delay of gratification was significant (direct effect = -.49 ). The perceived possibility of getting a future reward fully mediated the relation between the Future-Negative time perspective and the delay of gratification. Results indicated that the indirect effect was estimated to be between -.204 and -.022 with $95 \%$ confidence not containing 0 , confirming that the perceived possibility of getting a future reward did act as a mediator.

\section{Discussion}

This study investigated how an individual's TP affects the delay of gratification, using the construal level theory. To this end, the participants completed the Korean version of the S-ZTPI, as well as the temporal discounting task. Afterward, their psychological distance toward the future and perceived possibility of getting a future reward were measured using the visual analogue scale.

First of all, we consider that TP has a significant impact on the delay of gratification given that time is an important factor when assessing the value of a reward when there is a choice between two options. ${ }^{20}$ On the basis of results from prior research, we hypothesized a causal link between the time perspectives and the delay of gratification. ${ }^{13-15}$ We assumed that among the various TPs, Present-Hedonistic and Futureoriented (whether it is negative or positive) would have an 
impact on the delay of gratification. ${ }^{21,22}$ The results of this study showed that with the increase in the Present-Hedonistic and Future-Negative, the ability to delayed gratification decreases. In contrast, with the increase in the FuturePositive, the ability to delayed gratification increases. Therefore, hypotheses 1 and 2 were supported.

These results seem to be consistent with previous studies, which showed Present-Hedonistic is negatively associated with the delay of gratification, and that Futureoriented time perspective is positively associated with the delay of gratification. ${ }^{14,15}$ Zimbardo and Boyd have insisted on the delay of gratification as the essence of future time perspective and individuals with a high Present-Hedonistic time perspective are careless about future consequences of their actions. ${ }^{1}$ Also, according to a study examining the relationship between TP and risk-taking propensity, the strongest association was found between Present-Hedonistic and risktaking propensity among the various TPs, and futureorientation was linked with lower risk propensity. ${ }^{51}$ However, Future-Negative involves anticipating a negative outcome, so individuals who have high Future-Negative will pursue the smaller and sooner reward rather than the larger and later ones. ${ }^{4}$ In conclusion, these findings suggest that the subTPs could play different roles in the delay of gratification. Individuals who have high Present-Hedonistic and FutureNegative are more likely to suffer from a deficit in the delay of gratification, and individuals who have high Future-Positive are more likely to be successful in being able to delay gratification.

Although not included in our hypothesis, there is a point to note. The results demonstrated that Past-Negative has the second-largest impact on the delay of gratification and PastPositive also predicted the delay of gratification. According to Göllner, Ballhausen, Kliegel, and Forstmeier's research, people with a strong future orientation and without past orientation were the best in the delay of gratification, followed by people in the time expansive category with both future and past orientation. ${ }^{29}$ Bembenutty and Karabenick also suggested that a time perspective that is future-oriented or at least considers both past and future could improve the ability to delay gratification. ${ }^{15}$ However, these results have not been replicated by rigorous studies and no research has explored the mechanism of the relationship, to our knowledge. Thus, more research focusing on the past-oriented time perspective is needed.

This study also tried to explain the mechanism between TP and delayed gratification, using psychological distance and perceived possibilities as factors that affect the relationship with TP and delayed gratification. In the correlation analysis and meditation analysis, hypothesis 3 was not supported. The psychological distance was not associated with any TPs nor delayed gratification except those measured for 9 months. The psychological distance for 9 months seems to explain the delay of gratification well, consistent with the TP's direction. As preceding studies have explained feeling that the future is remote in Present-Hedonistic is consistent with having only slight regard for risk, or no concern for future consequences. ${ }^{1,3}$ Also, feeling that the future is near in Future-Positive is consistent with placing emphasis on future goals and individuals who think negatively about the future may have felt that the future is remote since people want to stay away from the negative one. ${ }^{52}$ According to the results, individuals with high Present-Hedonistic and Future-Negative will devaluate future rewards because they feel the future is remote. On the other hand, individuals with high Future-Positive will consider the future reward to be worth the wait, because they feel the future is close. Consequently, Present-Hedonistic and FutureNegative are more likely to fail in the delay of gratification and FuturePositive will succeed. However, these results alone are insufficient to prove the link among the TP, psychological distance, and delay of gratification, and well-designed additional study is needed. One plausible possibility is that we measured the psychological distance from 0 to 100 on the VAS scale while the perceived psychological distance is usually measured in multiple dimensions. ${ }^{53}$

Interestingly, the present findings suggest that the perceived possibility of getting a future reward plays an important role rather than the psychological distance toward the future. The higher the Present-Hedonistic time perspective, the lower the perceived possibility of getting a future reward, resulting in a failure to delayed gratification. On the other hand, the high Future-Positive time perspective did not increase the perception that rewards could be given in the future. This result suggests that the delay of gratification may be possible simply by thinking positively about the future. The perceived possibility of getting a future reward fully mediated the relation between Future-Negative and the delay of gratification. It means that individuals with Future-Negative time perspective fail to delay the gratification only when they feel that they cannot be rewarded in the future. As Carelli, Wiberg, and Wiberg have explained, the characteristic of Future-Negative involves thinking about the future with worry and, anticipating negative outcomes. ${ }^{4}$ Future-Negative time perspective directly reduces the perception that I can be rewarded, making the present reward preferred to the future reward. Therefore, we need to intervene differently for people with different time 
perspectives when intervening to increase the delay of gratification.

This study has several limitations. First, despite the rationale for the relationship between TP and psychological distance, no relationship was found in this study. Future research is needed since only the psychological distance measured for 9 months showed a significant correlation. Second, although the relevance between TP and perceived possibility of getting a future reward, the present findings cannot ensure the implicit relationships between TP and perceived possibility of getting a future reward as the questionnaire to measure psychological distance was self-reported. Thus, future research should more objectively examine these relationships using a measurement instrument like an implicit association test. Third, although we selected money as the reward for the delay of gratification, there can be different abilities to delayed gratification depending on the reward. In future studies, researchers should use diverse stimuli as rewards, such as food and alcohol. Finally, the participants were a nonclinical sample and were undergraduate students. Future research should make use of clinical samples in order to expand the present findings.

\section{Conclusion}

In conclusion, we investigated how an individual's TP affects the delay of gratification, using the psychological distance and the perceived possibility of getting a future reward. Our findings can help explain the mechanism by which the TP affects the delay of gratification. Importantly, our findings have implications for preventing the inability to delayed gratification. According to our results, problems associated with the delay of gratification such as smoking, addiction, and binge eating are more likely to occur in individuals with high PresentHedonistic and Future-Negative and low Future-Positive time perspectives. And it was suggested that the perceived possibility of getting a future reward is important in this relationship. Therefore, it is important to modify the perceived possibility of getting a future reward in order to prevent problems like addiction and binge eating in persons with high Present-Hedonistic and FutureNegative time perspectives.

\section{Funding}

This research was supported by the Ministry of Education of the Republic of Korea and the National Research Foundation of Korea (NRF-2013S1A5A2A01019660).

\section{Disclosure}

Hyo-Jeong Kim reports grants from the Ministry of Education of the Republic of Korea and the National Research Foundation of Korea, during the conduct of the study. The authors report no other potential conflicts of interest in this work.

\section{References}

1. Zimbardo P, Boyd J. The Time Paradox: The New Psychology of Time That Will Change Your Life. Simon and Schuster; 2008.

2. Zimbardo PG, Boyd JN. Putting time in perspective: a valid, reliable individual-differences metric. In: Time Perspective Theory; Review, Research and Application. Springer; 2015:17-55.

3. Zimbardo PG, Boyd JN. Putting time in perspective: a valid, reliable individual-differences metric. J Pers Soc Psychol. 1999;77 (6):1271-1288. doi:10.1037/0022-3514.77.6.1271

4. Carelli MG, Wiberg B, Wiberg M. Development and construct validation of the Swedish Zimbardo time perspective inventory. Eur $J$ Psychol Assess. 2011;27(4):220-227. doi:10.1027/1015-5759/ a000076

5. Boyd J, Zimbardo P. Time perspective health and risk taking. In: Strahman A, Joireman J, editors. Understanding Behavior in the Context of Time: Theory, Research and Applications. Mahwah, NJ: erlbaum; 2005.

6. Boniwell I, Osin E, Alex Linley P, Ivanchenko GV. A question of balance: time perspective and well-being in British and Russian samples. J Posit Psychol. 2010;5(1):24-40. doi:10.1080/17439760 903271181

7. Zhang JW, Howell RT, Stolarski M. Comparing three methods to measure a balanced time perspective: the relationship between a balanced time perspective and subjective well-being. Happiness Stud. 2013;14(1):169-184. doi:10.1007/s10902-0129322-x

8. Drake L, Duncan E, Sutherland F, Abernethy C, Henry C. Time perspective and correlates of wellbeing. Time Soc. 2008;17 (1):47-61. doi:10.1177/0961463X07086304

9. Verdejo-García A, Lawrence AJ, Clark L. Impulsivity as a vulnerability marker for substance-use disorders: review of findings from high-risk research, problem gamblers and genetic association studies. Neurosci Biobehav Rev. 2008;32(4):777-810. doi:10.1016/j. neubiorev.2007.11.003

10. Hodgins DC, Engel A. Future time perspective in pathological gamblers. J Nerv Ment Dis. 2002;190(11):775-780. doi:10.1097/ 00005053-200211000-00008

11. Keough KA, Zimbardo PG, Boyd JN. Who's smoking, drinking, and using drugs? Time perspective as a predictor of substance use. Basic Appl Soc Psych. 1999;21(2):149-164. doi:10.1207/S15324834BA 210207

12. Daugherty JR, Brase GL. Taking time to be healthy: predicting health behaviors with delay discounting and time perspective. Pers Individ Dif. 2010;48(2):202-207. doi:10.1016/j.paid.2009.10.007

13. Teuscher U, Mitchell SH. Relation between time perspective and delay discounting: a literature review. Psychol Rec. 2011;61 (4):613-632. doi:10.1007/BF03395780

14. Romer D, Duckworth AL, Sznitman S, Park S. Can adolescents learn self-control? Delay of gratification in the development of control over risk taking. Prev Sci. 2010;11(3):319-330. doi:10.1007/s11121-0100171-8

15. Bembenutty $\mathrm{H}$, Karabenick SA. Inherent association between academic delay of gratification, future time perspective, and self-regulated learning. Educ Psychol Rev. 2004;16(1):35-57. doi:10.1023/B:EDPR.0000012344.34008.5c 
16. Avci S. Relations between self regulation, future time perspective and the delay of gratification in university students. Education. 2013;133 (4):525-537.

17. Mischel W, Underwood B. Instrumental ideation in delay of gratification. Child Dev. 1974;45(4):1083-1088. doi:10.2307/1128 098

18. Mischel W, Ayduk O. Self-regulation in a cognitive-affective personality system: attentional control in the service of the self. Self Identity. 2002;1(2):113-120. doi:10.1080/152988602317319285

19. Reynolds B, Schiffbauer R. Delay of gratification and delay discounting: a unifying feedback model of delay-related impulsive behavior. Psychol Rec. 2005;55(3):439-460. doi:10.1007/BF03395520

20. Wittmann M, Leland DS, Paulus MP. Time and decision making: differential contribution of the posterior insular cortex and the striatum during a delay discounting task. Exp Brain Res. 2007;179 (4):643-653. doi:10.1007/s00221-006-0822-y

21. Beenstock J, Adams J, White M. The association between time perspective and alcohol consumption in university students: cross-sectional study. Eur J Public Health. 2011;21(4):438-443. doi:10.1093/eurpub/ckp225

22. Cosenza M, Ciccarelli M, Nigro G. The steamy mirror of adolescent gamblers: mentalization, impulsivity, and time horizon. Addict Behav. 2019;89:156-162. doi:10.1016/j.addbeh.2018.10.002

23. Nigro G, Cosenza M, Ciccarelli M. The blurred future of adolescent gamblers: impulsivity, time horizon, and emotional distress. Front Psychol. 2017;8:486. doi:10.3389/fpsyg.2017.00486

24. Petry NM, Bickel WK, Arnett M. Shortened time horizons and insensitivity to future consequences in heroin addicts. Addiction. 1998;93(5):729-738. doi:10.1046/j.1360-0443.1998. 9357298.x

25. Shafikhani M, Bagherian F, Shokri O. The mediating role of time perspective in the relationship between general self-efficacy and the tendency toward substance abuse in female adolescents. Int J Psychol. 2018;12(1):208-231. doi:10.24200/ ijpb.2018.58149

26. Cosenza M, Griffiths MD, Nigro G, Ciccarelli M. Risk-taking, delay discounting, and time perspective in adolescent gamblers: an experimental study. J Gambl Stud. 2017;33(2):383-395. doi:10.1007/ s10899-016-9623-9

27. Chavarria J, Allan NP, Moltisanti A, Taylor J. The effects of present hedonistic time perspective and past negative time perspective on substance use consequences. Drug Alcohol Depend. 2015;152:39-46. doi:10.1016/j.drugalcdep.2015.04.027

28. Nuttin J. Future Time Perspective and Motivation: Theory and Research Method. Psychology Press; 2014.

29. Göllner LM, Ballhausen N, Kliegel M, Forstmeier S. Delay of gratification, delay discounting and their associations with age, episodic future thinking, and future time perspective. Front Psychol. 2018;8 (2304). doi:10.3389/fpsyg.2017.02304

30. Brooks M, Miller E, Abebe K, Mulvey E. The observed longitudinal relationship between future orientation and substance use among a cohort of youth with serious criminal offenses. Subst Use Misuse. 2018;53(12):1925-1936. doi:10.1080/10826084.2018.1441311

31. Mischel W, Baker N. Cognitive appraisals and transformations in delay behavior. $J$ Pers Soc Psychol. 1975;31(2):254-261. doi:10.1037/h0076272

32. Mazur JE. Quantitative analyses of behavior: the effect of delay and of intervening events on reinforcement value. In: An Adjusting Procedure for Studying Delayed Reinforcement. NJ: Hillsdale; 1987:55-73.

33. Trope Y, Liberman N. Construal-level theory of psychological distance. Psychol Rev. 2010;117(2):440-463. doi:10.1037/a0018 963
34. Liberman N, Trope Y. The role of feasibility and desirability considerations in near and distant future decisions: a test of temporal construal theory. J Pers Soc Psychol. 1998;75(1):5-18. doi:10.1037/ 0022-3514.75.1.5

35. Trope Y, Liberman N. Temporal construal. Psychol Rev. 2003;110 (3):403-421. doi:10.1037/0033-295X.110.3.403

36. Benoit RG, Gilbert SJ, Burgess PW. A neural mechanism mediating the impact of episodic prospection on farsighted decisions. J Neurosci. 2011;31(18):6771-6779. doi:10.1523/JNEUROSCI.6559-10.2011

37. Peters J, Büchel C. Episodic future thinking reduces reward delay discounting through an enhancement of prefrontal-mediotemporal interactions. Neuron. 2010;66(1):138-148. doi:10.1016/j.neuron.20 10.03.026

38. Stolarski M, Bitner J, Zimbardo PG. Time perspective, emotional intelligence and discounting of delayed awards. Time Soc. 2011;20 (3):346-363. doi:10.1177/0961463X11414296

39. Macaskill AC, Hunt MJ, Milfont TL. On the associations between delay discounting and temporal thinking. Pers Individ Dif. 2019;141:166-172. doi:10.1016/j.paid.2019.01.007

40. Estle SJ, Green L, Myerson J, Holt DD. Discounting of monetary and directly consumable rewards. Psychol Sci. 2007;18(1):58-63. doi:10.1111/j.1467-9280.2007.01849.x

41. MacKillop J, Miller JD, Fortune E, et al. Multidimensional examination of impulsivity in relation to disordered gambling. Exp Clin Psychopharmacol. 2014;22(2):176-185. doi:10.1037/a0035874

42. O'Connell G, Christakou A, Haffey A, Chakrabarti B. The role of empathy in choosing rewards from another's perspective. Front Hum Neurosci. 2013;7:174.

43. Cheong K, Kim J. Effects of reward magnitude on the performance of delayed discounting task: focusing on smoking and drinking behaviors. Korean J Clin Psychol. 2013;32(1):55-76. doi:10.15842/ kjcp.2013.32.1.004

44. Johnson MW, Bickel WK. Within-subject comparison of real and hypothetical money rewards in delay discounting. J Exp Anal Behav. 2002;77(2):129-146. doi:10.1901/jeab.2002.77-129

45. Madden GJ, Begotka AM, Raiff BR, Kastern LL. Delay discounting of real and hypothetical rewards. Exp Clin Psychopharmacol. 2003;11(2):139-145. doi:10.1037/1064-1297.11.2.139

46. Patton JH, Stanford MS, Barratt ES. Factor structure of the Barratt impulsiveness scale. J Clin Psychol. 1995;51(6):768-774. doi:10.1002/1097-4679(199511)51:6<768::AIDJCLP2270510607>3.0.CO;2-1

47. Aiken LS, West SG, Reno RR. Multiple Regression: Testing and Interpreting Interactions. Sage; 1991.

48. Fox J, Long JS. Modern Methods of Data Analysis. Newbury Park, CA: Sage Publications; 1990.

49. Myerson J, Green L, Warusawitharana M. Area under the curve as a measure of discounting. Exp Anal Behav. 2001;76(2):235-243. doi:10.1901/jeab.2001.76-235

50. Hayes AF. Introduction to Mediation, Moderation, and Conditional Process Analysis: A Regression-Based Approach. Guilford Press; 2013.

51. Jochemczyk Ł, Pietrzak J, Buczkowski R, Stolarski M, Markiewicz Ł. You only live once: present-hedonistic time perspective predicts risk propensity. Pers Individ Dif. 2017;115:148153. doi:10.1016/j.paid.2016.03.010

52. Mühlberger A, Neumann R, Wieser MJ, Pauli P. The impact of changes in spatial distance on emotional responses. Emotion. 2008;8(2):192-198. doi:10.1037/1528-3542.8.2.192

53. Chen HS, Mitchell RK, Brigham KH, Howell R, Steinbauer R. Perceived psychological distance, construal processes, and abstractness of entrepreneurial action. J Bus Ventur. 2018;33(3):296-314. doi:10.1016/j.jbusvent.2018.01.001 


\section{Publish your work in this journal}

Psychology Research and Behavior Management is an international, peer-reviewed, open access journal focusing on the science of psychology and its application in behavior management to develop improved outcomes in the clinical, educational, sports and business arenas. Specific topics covered in the journal include: Neuroscience, memory and decision making; Behavior modification and management; Clinical applications; Business and sports performance management; Social and developmental studies; Animal studies. The manuscript management system is completely online and includes a very quick and fair peer-review system, which is all easy to use. Visit http://www. dovepress.com/testimonials.php to read real quotes from published authors. 